THE JOURNAL OF TEACHING ENGLISH FOR SPECIFIC AND ACADEMIC PURPOSES

Vol. 9, No 4, Special Issue, 2021, pp. 575-586

UDC: 811.111 '255.4 https://doi.org/10.22190/JTESAP2104575K

Original scientific paper

\title{
DIGITAL TEACHER FEEDBACK AS A TRANSLATION ASSESSMENT TOOL
}

Tetiana Korol

Poltava University of Economics and Trade, Ukraine

\begin{abstract}
This paper analyses the opportunities of digital feedback delivery, its use in the translation classroom, and its application as the tool of active instruction and formative assessment at university level. A mixed research design involved 33 third-year students of Poltava University of Economics and Trade majoring in Translation. They participated in the study voluntarily in the spring term of 2019/2020 academic year. The effectiveness of the digital teacher feedback of different modality was estimated with the help of a questionnaire from two perspectives: students' behavioral engagement associated with feedback convenience in use for translation revisions and their affective engagement concerning emotional saturation of the suggested type of digital feedback. Students' preferences were collated with their results in leading sensory channel test and acquired translation competency level. According to the received data, overwhelming $69.7 \%$ of undergraduate translators appeared to be digitals as per their leading sensory channel, which had no statistically significant impact on the preferred feedback modality inversely to students' translation competency level. The observed correlation proved the viability of both suggested digital feedback modalities at different stages of the training process. It was concluded that digital teacher feedback promotes the development of the students' translation skills in particular and leverages formative assessment practices in the translation classroom in general.
\end{abstract}

Key words: digital teacher feedback, translation assessment, undergraduate translators, leading sensory channel

\section{INTRODUCTION}

Information technologies have steadily proliferated in all the spheres of modern society over the last decade, and education is not an exception. Both teachers and students should be prepared to use varied general and application software in order to facilitate instruction process and improve their training and learning outcomes. That is why, progressive language and translation teachers keep searching for the efficient digital tools to enhance their teaching and assessment practices on the one hand, and diversify their students' expertise in information technologies, valuable from the viewpoint of their prospective professional activity on the other. An effective digital tool can spare teacher's time and efforts and provide an efficient approach to students' learning, especially in

Submitted March 23 $3^{\text {rd }}, 2021$, accepted for publication August $1^{\text {st }}, 2021$

Corresponding author: Tetiana Korol. Poltava University of Economics and Trade, Ukraine.

E-mail: koroltatyanalq@gmail.com

(C) 2021 by University of Niš, Serbia | Creative Commons License: CC BY-NC-ND 
terms of translation training. According to the provisions of social constructivist approach (Kiraly, 2005), it should be focused on the development of students' translation skills through and by doing due to the continuous interaction of all the participants involved into the translation process.

Feedback is considered one of indispensable components of translation performance. It naturally comes in the form of editor's or project manager's remarks in real professional world and teacher's or peer's comments and suggestions in educational setting. In case of professional translation, it tends to be delivered with the help of a digital note option, commonly available in different computer-aided translation tools, widely used in modern translation industry. This fact combined with the current trends in offline, online and/or blended university training urges translation teachers to master and employ digital feedback on translations performed by their students in order to convert it into a powerful tool of formative assessment.

Having specified feedback definition by Hattie and Timperley (2007) within our research, we view digital teacher feedback as any information supplied by a teacher to a student as to particular aspects of their translation performance generated with the help of any appropriate software and delivered in digital mode (written, audio- or video-recorded).

Compared to live oral or handwritten one, digital feedback features a number of obvious benefits: 1) it gives the opportunity to take into account individual needs of undergraduate translators with different learning styles and translation competency levels in terms of varied presentation modality; 2) it allows teachers to simulate real-life professional situations of interaction between different participants of a translation project; 3 ) it promotes timely comment delivery, its optimal storage and subsequent retrieval of its content if the need arises; 4) it enables teachers to provide more detailed, emotionally saturated and personalized comment on a particular translation product; 5) it spares teacher's time and efforts due to easy generation and delivery procedures. However, according to the conducted literature review (presented below) modern translation pedagogy lacks relevant research devoted to the opportunities of digital teacher feedback use at university level.

Therefore, the objectives of this study are: 1) to determine and analyze some accessible and practical modes of digital feedback delivery in the translation classroom; 2) to define undergraduate translators' attitudes to written and audio digital teacher feedback in contrast to the paper handwritten one; 3) to outline the application peculiarities of digital feedback of different modality for translation assessment from the viewpoint of students' leading sensory channels and their acquired translation competency levels.

\section{LITERATURE REVIEW}

In modern applied linguistics feedback utilization is examined extensively in terms of foreign language productive skills development (Lim and Phua, 2019; Tsagari, 2019; Zarei and Rezadoust, 2020), while its study in the translation classroom is rather scarce and limited to certain aspects only. For example, Južnič (2013) compared feedback perception by translation trainers and trainees at Slovenian universities. Alfayyadh (2016) contrasted the functions performed by translation feedback in different national educational environments. Neunzig and Tanqueiro (2005) summarized the distinguishing features of a translation feedback automatically generated by a computer. Washbourne (2014) considered written feedback on students' translation performance from the 
viewpoint of dialogic and iterative approaches. Pietrzak (2014) presented feedback circulation model within a group of translation students. Zheng, Zhong, Yu and Li (2020) examined the correlation between students' translation competency level and their engagement with the received teacher feedback. Finally yet importantly, Yu, Zhang, Zeng and Lin (2019) dealt with the teacher's strategies for written translation feedback generation and their impact upon students' revision.

As far as we can see, modern translation pedagogy completely lacks the studies directed to the investigation of digital feedback use, which have sprung recently in teaching foreign language writing and other creative activities. Their intensification is closely connected with the development and growing accessibility of a wide range of free computer software that allows teachers to generate multimodal digital feedback. To date available types of digital feedback include: 1) electronic written, both in-text (with the help of commenting and editing features of offline and online word processors such as Microsoft Word or Google Docs which provide the opportunity to track changes and corrections made by a teacher and add some text bubbles) and out-of-text (in the form of emails and other text messages as well as blog posts and online chats); 2) recorded audio (due to built-in features of Turnitin or Canvas online platforms and Google Docs plugins such as Kaizena or Chrome extensions, namely Read\&Write and Mote); 3) recorded video (captured with the use of screencasting software including Screencastify, Screencast-o-matic, etc.).

According to McCarthy (2015), video feedback appeared to be more engaging and easier for students to comprehend and use compared to written and audio ones within summative assessment at university level. Similar results were received by Henderson and Phillips (2015), although some drawbacks of this feedback modality were distinguished, namely the difficulty of matching global video-comment to the particular faults of text-based assignments and students' anxiety about watching personalized video feedback. Some technical issues connected with operating system requirements, optimal volume or quality of the received video file and its sharing/storing arose as well.

Having analysed the studies, devoted to the investigation of the students' attitude to audio feedback recorded in the form of MP3 files within teaching and assessing different aspects of English writing (Cavanaugh and Song, 2014; Dalton, 2018), drama studies (Pearson, 2018) as well as bioscience written assignments (Voelkel and Mello, 2014) we have singled out some of its common benefits. Audio feedback appears to be clearer and less ambiguous to the students due to its length and abundance compared to the written one. It seems more comprehensive and accessible than the written one. This modality of feedback delivery ensures its emotional saturation. Audio feedback sounds supportive and motivating to its recipients. It appears to be individual and personalized. It leads to future strategizing and feed-forwarding. Audio feedback appeals to teachers, promoting their self-efficiency beliefs and converting assessment into mutually engaging process.

However, audio feedback research raised some kind of discrepancies and contradictions as well. Its generation and use can be technically challenging to both teachers and students. There is no evidence that it leads to students' higher performance and has impact that is more positive on their learning outcomes. Producing audio feedback teachers tend to comment on global issues of assignment performance rather than provide a detailed report of its strengths and weaknesses. A need for noting down teacher's corrections and suggestions can create additional obstacles to proper assignment revision by the students.

Anyway, the obvious functionality and growing accessibility of this type of digital feedback appears to be promising in the context of formative translation assessment and requires thorough examination at university level. 


\section{METHOD}

A mixed research design has been adopted for this study. Its qualitative aspect was concentrated on the collection of two sets of data about: 1) the students' attitudes to digital feedbacks of different modalities from the perspectives of their behavioral and affective engagement; 2) the participants' leading sensory channel.

Students' behavioral engagement is connected with feedback functionality that provides sufficient readiness to revise their translations in line with the received teacher comments. While their affective engagement concerns individual emotional response and attitude to the suggested type of digital feedback and its emotional saturation (Zheng, Zhong, Yu and Li, 2020).

In its turn, research quantitative aspect dealt with the statistical analysis of the correlation of the students' preferred digital feedback modality with their leading sensory channel and acquired competency level. On this basis, the list of the criteria to select software for translation feedback generation was drafted and the ways to apply different modes of digital feedback were defined. They allowed us to personalize translation training and assessment to some extent.

The research was carried out in the spring term of 2019-2020 academic year at Poltava University of Economics and Trade. In total, 33 third-year students (25 women and 8 men), aged from 19 to 23, majoring in Translation, voluntarily participated in this survey. They have been taking Translation Practice course for a year, have acquired particular translation competency level and were used to receiving and utilizing handwritten teacher feedback on their full translation performance. For the sake of our survey, the students were subdivided into three groups of translation competency according to their course learning outcomes: high (14 students), medium (12 students) and low (7 students). Participants' demographic information is summarized in Table 1.

Table 1 Research participants' demographic information

\begin{tabular}{|c|c|c|c|c|c|c|c|c|c|c|c|c|}
\hline \multicolumn{3}{|c|}{ Gender } & \multicolumn{6}{|c|}{ Age } & \multicolumn{4}{|c|}{ Translation competency level } \\
\hline \multicolumn{2}{|c|}{ Male } & Female & 19 & 20 & \multicolumn{2}{|c|}{21} & \multicolumn{2}{|c|}{23} & High & \multicolumn{2}{|c|}{ Medium } & Low \\
\hline 8 & $24 \%$ & \begin{tabular}{l|l|}
25 & $76 \%$ \\
\end{tabular} & \begin{tabular}{|l|l|}
13 & $39 \%$ \\
\end{tabular} & \begin{tabular}{l|l|}
16 & $49 \%$ \\
\end{tabular} & 3 & $9 \%$ & 1 & $3 \%$ & \begin{tabular}{|l|l|}
14 & $43 \%$ \\
\end{tabular} & 12 & $36 \%$ & \begin{tabular}{l|l|}
7 & $21 \%$ \\
\end{tabular} \\
\hline & 33 & $100 \%$ & 3. & & & & $0 \%$ & & 33 & & & $00 \%$ \\
\hline
\end{tabular}

During the term, the third-year students had to perform and submit one full translation of English popular science article in Business Administration into Ukrainian per week. Their translations were assessed and reflected on by the teacher on a regular basis. Translations 1-8 were followed with the electronic written in-text feedback generated with the help of built-in Google Docs commenting and editing feature (see Fig. 1), while translations 9-16 received recorded audio feedback created with the use of Chrome extension Mote (see Fig. 2). 


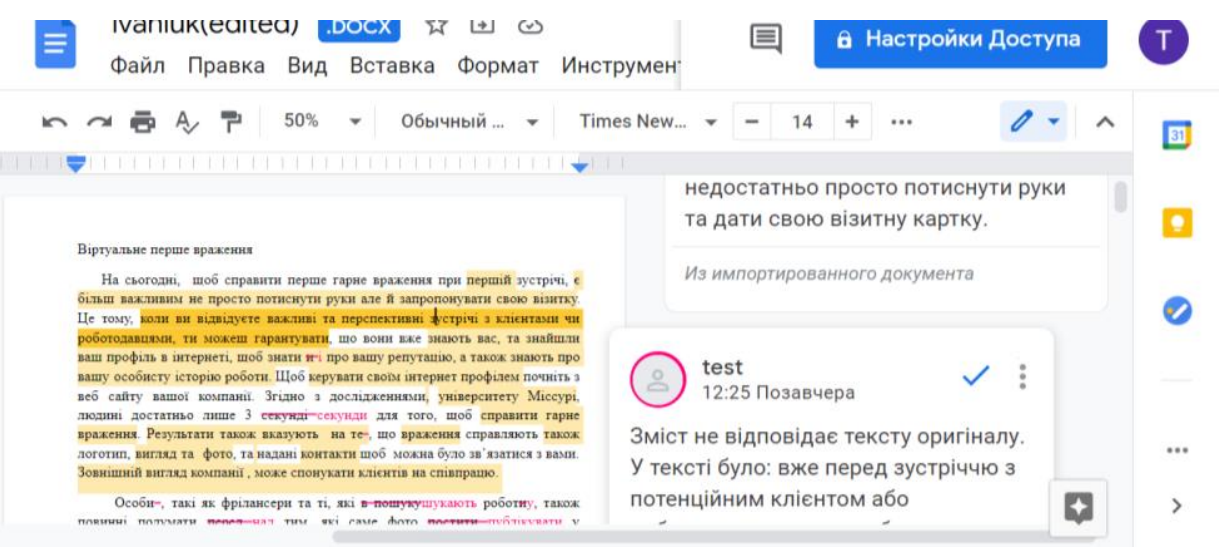

Fig. 1 Sample view of a translation with written digital feedback

In order to achieve a desired formative effect, the students were asked to revise and resubmit their translations, corrected according to the obtained feedback. The observation of their behavioral patterns connected with the feedback analysis and use was carried out. At the end of the term a questionnaire to study their opinion on feedback in general and its modality in particular was developed and applied. It contained 12 close-ended questions presented with the help of Google forms.

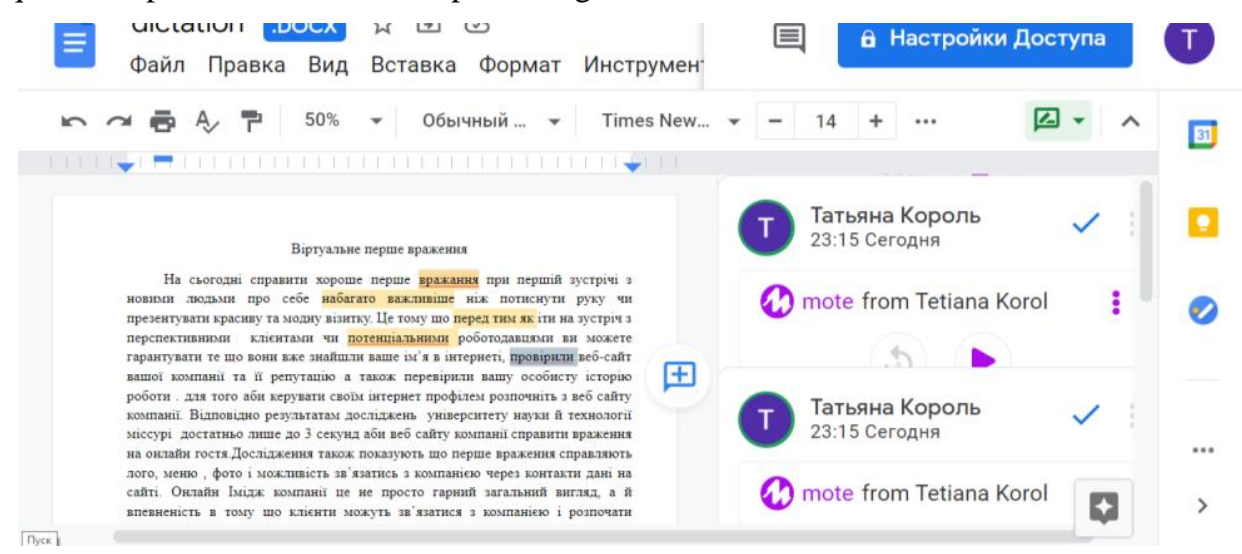

Fig. 2 Sample view of a translation with audio digital feedback

In average, it took about 9-12 minutes to complete the following survey online:

1. How do you evaluate the importance of feedback in efficient translation training?

a) Extremely important

b) Important

c) Relatively important

d) Not so important

e) Not important at all

2. What type of feedback delivery do you consider more appropriate?
a) Individual
b) Group 
3. Did you feel any difference between paper and digital feedback?

a) Yes, and it was huge

b) Yes, but it was slight

c) No

4. Did you feel any difference between written and audio digital feedback?

a) Yes, and it was huge

b) Yes, but it was slight

c) No

5. Which type of feedback delivery is more convenient for further translation revision and correction?

a) Handwritten

b) Written digital

c) Audio digital

6. Which type of feedback delivery appeared to be more motivating and engaging to you?
a) Handwritten
b) Written digital
c) Audio digital

7. What are the main disadvantages of a written digital feedback? More than one answer can be chosen.

a) It is not easy to process and use

b) It does not always provide sufficient information on the required /made corrections

c) It does not help understand teacher's attitude to my translation

d) It does not motivate me to improve my translation skills

e) It does not appear to be personal and engaging enough

f) It is too boring and monotonous to read and use

8. What are the main disadvantages of an audio digital feedback? More than one answer can be chosen.

a) It is not easy to process and use

b) It does not always provide sufficient information on the required /made corrections

c) It does not help understand teacher's attitude to my translation

d) It does not motivate me to improve my translation skills

e) It does not appear to be personal and engaging enough

f) It is too boring and monotonous to listen to and use

9. What are the main advantages of a written digital feedback? More than one answer can be chosen.

a) It is fast to process and easy to use

b) It provides extensive information on the required /made corrections

c) It reflects teacher's attitude to my translation

d) It motivates me to revise my translation and improve my translation skills

e) It always appears to be personal and makes me feel connected

f) It reproduces the emotional background of real-life interaction

10. What are the main advantages of audio digital feedback? More than one answer can be chosen.
a) It is fast to process and easy to use
b) It provides extensive information on the required /made corrections
c) It reflects teacher's attitude to my translation
d) It motivates me to improve my translation skills
e) It always appears to be personal and makes me feel connected
f) It reproduces the emotional background of real-life interaction 
11. What makes an ideal digital feedback to you? More than one answer can be chosen.

a) It should be fast to process and easy to use

b) It should provide extensive information on the required /made corrections

c) It should reflect teacher's attitude to my translation

d) It should motivate me to revise my translation

e) It should be personal and addressed to me only

f) It should reproduce real-life interaction and communication with the teacher and be emotionally saturated

12. Does feedback delivery type influence the quality of your translation revision and correction?

a) Yes, fully

b) Yes, partially

c) No

The received data were complemented with the results of psychological diagnostic test for leading sensory channel determination (Lobanov, 2004). This test contained 12 questions with 4 possible answers each. The students were asked to rate them from 1 to 4 , where 1 denoted their least typical behavior pattern while 4 corresponded to the most typical one. Test results were interpreted according to the standardized key, which distinguished between the four sensory channels (auditory, visual, kinesthetic, and digital) and allowed us to determine the leading one for each survey participant. Finally, students' leading sensory channels, current translation competency levels and preferred feedback modality were collated, while their correlation was verified statistically.

\section{RESULTS}

The conducted survey of 33 third-year students majoring in Translation has revealed that the majority of them $(87.88 \%)$ find feedback extremely important for the efficient translation training. Even more students $(96.97 \%)$ consider individual feedback delivery more appropriate and viable, and only one participant feels completely satisfied with impersonal group feedback. It is worth noting here, that this student demonstrated low translation competency level at the end of the course.

All the respondents $(100 \%)$ experienced either huge $(87.88 \%)$, or slight $(12.12 \%)$ difference between the paper (handwritten) and digital feedback. The students with high translation competency level (11 out of 14) tended to notice insufficient difference between the written and audio digital feedback, while most of the students with medium and low translation competency levels (11 out of 12 and 7 out of 7 respectively) felt a great difference between them.

Most of the students $(51.52 \%)$ consider audio digital feedback delivery more convenient for further translation revision and correction. One third of the respondents $(30.30 \%)$ preferred written digital feedback use for this purpose and only $18.18 \%$ of them insisted on handwritten feedback convenience. In all students' opinion (100\%), audio digital feedback appeared to be more motivating and engaging compared to other modalities.

To the participants' mind, the leading drawbacks of written digital feedback include: 1) lack of some sufficient information on the required changes in the target text or corrections made $(84.85 \%)$. It can be explained with time and space constraints encountered by any 
teacher in case of this type of feedback delivery; 2) no opportunity to decode real teacher's positive or negative attitude to the translation performed $(72.73 \%)$ because of predominantly emotionally neutral written digital feedback and its main focus on the straightforward information presentation; 3) low motivational potential of such feedback type (69.7\%) connected with the absence of real opportunity to encourage students explicitly; 4) its boring and monotonous presentation $(60.6 \%)$ caused with the fact that any teacher tends to use typical structures and expressions to comment on common students' mistakes and errors, especially in case of brief texting. Overwhelming benefit of this feedback delivery acknowledged by the majority of the surveyed $(100 \%)$ was simplicity in perception and processing as well as convenience in practical application.

As for audio digital feedback, more than a half of the students $(54.55 \%)$ complained of the only problem connected with the inconvenience of its analysis and use. Indeed, audio feedback utilization can require recurrent listening and even note-taking to be viable and effective. However, this type of feedback delivery has plenty of obvious advantages according to the students. Firstly, it provides extensive information on what should be done to correct translation mistakes and avoid them in the future $(69.7 \%$ of students). About $96.97 \%$ of the respondents was sure that it clearly reflected teacher's real attitude to their translation performance due to the opportunity to hear his/her voice and intonation. Almost $90.9 \%$ of the undergraduate translators admitted that it was rather motivating and promoted their translation revision and further translation skill improvement. Due to audio feedback delivery, $72.73 \%$ of the students felt engaged and treated teacher's comments as highly personal ones. Finally, $81.82 \%$ of the participants enjoyed the reproduced emotional aspect of real-life communication and interaction with their teacher provided by audio digital feedback.

From behavioral aspect, the ideal feedback delivery looks like this: 1) convenient to analyse and process, easy to use (100\% of the respondents); 2 ) provides all the sufficient information on the required corrections (100\% of the survey participants); 3) highly motivational, encourages to revise / correct the translation and to develop students' translation skills $(90.9 \%)$. At the same time affective engagement appeared to be extremely important from the respondents' viewpoint as well. From this perspective, an ideal feedback should: 1) clearly reflect teacher's attitude to current translation performance ( $100 \%$ of the students); 2 ) be personalized and engaging (100\% of the surveyed); 3) reproduce the emotional component of real-life interaction $(72.73 \%$ of the participants). Based on the conducted survey, we can claim that both behavioral and affective aspects of feedback delivery matter to the undergraduate translators and influence their translation revision activity in particular and translation skills development in general. All the students agreed that feedback modality affected their revision efficiency either greatly $(48.48 \%)$ or slightly $(51.52 \%)$.

According to the results of psychological test aimed at the determination of the students' leading sensory channel (Lobanov, 2004), overwhelming majority of the testees $(69.7 \%)$ belong to digitals. It means that they are able to perceive information instantly from diverse channels at a time and function efficiently within several modalities easily switching or combining them. The second most numerous category is kinesthetic students $(21.21 \%)$. Emotions, attitudes and direct interaction are their main sources of information and knowledge acquisition. Visual students (6.06\%) and auditory one (3.03\%) are the least represented among the surveyed undergraduate translators.

The statistical verification of the correlation of the students' leading sensory channel, translation competency level and preferred feedback modality carried out with the help of 
Gamma Coefficient in the software Statistica 10.0 showed: 1) statistically significant connection between the students' translation competency level and their preferred feedback modality $(0.677966$ at $\mathrm{p}<0.05) ; 2)$ no statistically significant relation between the students' leading sensory channel and their preferred feedback modality $(-0.045752$ at $\mathrm{p}<0.05)$. The students with low level of translation competency mostly went for handwritten feedback (4 students out of 7) and written digital one ( 3 out of 7). In most cases, the students with medium translation competency level chose digital feedback: audio (7 out of 12) and written (3 out of 12), although two of them still preferred handwritten one. Finally, the students with high competency level opted for digital feedback only: audio and written (10 and 4 participants respectively).

\section{DISCUSSION}

The obtained data prove the importance of feedback, especially individual one in translation training at university level, and highlight digital feedback delivery potential in this respect. This fully complies with the positive opinion on digital feedback of different modality previously fixed by other researchers in varied areas (Cavanaugh and Song, 2014; Dalton, 2018; McCarthy, 2015; Pearson, 2018; Tsagari, 2019; Voelkel and Mello, 2014).

The analysis of the research experience and survey results allowed us to draft the following list of criteria to select optimal software for audio digital feedback generation and delivery in the translation assessment: 1) it should be easy and completely free to install; 2) it should provide absolutely user-friendly interface; 3 ) it should have minimum system requirements; 3 ) its output should be accessible from any device and run without obligatory software installation; 4) it should enable teachers to produce records long enough to deliver extensive in-text comments (up to $90 \mathrm{sec}$.); 5) it should contain built-in option of transcript creation in different languages to assist in parallel generation of bilingual written digital feedback; 6) it should contain refback option to promote real-life interaction; 7) it should keep track of students' views and responses.

Audio digital feedback created with the help of optimal software provides translator trainees' behavioral and affective engagement equally important for the efficient translation revision and correction as found by Zheng, Zhong, Yu and $\mathrm{Li} \mathrm{(2020).} \mathrm{There} \mathrm{is}$ an assumption that teacher's feedback approach and strategies are also affected by its modality. As a result, audio feedback is more extensive and fluent, emotionally saturated, personalized and encouraging compared to a written digital one.

Finally, the fixed dominance of undergraduate translators with digital sensory channel supports general trends closely connected with upbringing peculiarities and lifestyle of current student generation in Ukraine. Synekop (2018) obtained similar results for more numerous population concerning leading sensory channels of future IT specialists. Kinesthetic students were the second-largest cohort in our research. These results can be partially caused by the prevalence of female participants or translator's occupational peculiarities and expectations. In the context of our study, this fact can also explain the equal significance of behavioral and affective aspects of feedback for the survey participants. At the same time, digital students' dominating number mitigated the modality impact on feedback viability in the translation classroom.

Strong correlation between the students' translation competency level and preferred feedback modality requires consecutive and situational involvement of handwritten, 
written and audio digital feedbacks at different stages of instruction and assessment in the translation classroom following and monitoring the process of translation competency acquisition. There is an idea to start translators' training with a handwritten teacher feedback on the performed translations, since it often involves a lot of different explicit corrections and ready-made translation solutions suggested by a teacher. Such feedback type is straightforward enough to promote efficient revision procedures in particular and direct the training process in general. The next training stage should employ written digital feedback, which is more convenient from the viewpoint of its generation and further use for both teachers and students. However, it can be too typical and impersonal because of the temptation to copy and paste some standard clichés and patterns in its preparation and presentation by the teacher. This feedback modality corresponds to more implicit comments combined with hinting through highlighting, guiding how to correct and prevent such mistakes and errors in the future. It is worth noting that such feedback strategy is more productive at the intermediate training stages. Finally, audio digital feedback reproduces some kind of real-life discussion and interaction. It helps motivate and encourage, challenge and praise students' particular translation solutions. Due to its emotional saturation, it appears to be engaging and personal. The analysis of such type of feedback for students is exciting itself. In case of handwritten and written digital feedbacks, everything is clear from the first sight, while in case of audio digital feedback there is always some unexpected mystery, which creates some cognitive discrepancy to be solved and overcome. So audio digital feedback is suitable for more implicit corrections and comments with multiple suggestions and flexible solutions to be evaluated and interpreted by the student in the process of further translation revision. Such feedback content and presentation are efficient enough with the students who have already acquired some basic translation skills. As we can see, digital feedback of different modalities provides opportunity to adjust feedback delivery and use to students' needs according to the training stage.

\section{CONCLUSION}

Current trends in education and translation industry require the adoption and active application of digital teacher feedback practices in the translation classroom due to their obvious benefits. Digital teacher feedback as any information supplied by a teacher to a student concerning particular aspects of their translation performance is generated with the help of appropriate software and delivered in digital written, audio- or video-recorded mode. In the context of modern digital society, written and audio digital feedbacks look extremely promising. Moreover, they are more affordable and functional compared to video digital one, especially in the form of electronic written in-text comments produced with the help of editing built-in features of word processors MS Word and Google Docs as well as audio in-text comments recorded with the help of such Chrome extensions as Mote or Read\&Write.

The conducted study provides the empirical evidence of digital teacher feedback efficiency in the translation classroom. According to the survey results, all the respondents experienced the difference between paper and digital feedbacks. More than $81 \%$ of the students preferred to receive a digital teacher feedback on their translations. All the survey participants stressed that digital feedback modality influenced their revision performance. In their opinion, the main advantage of a written digital feedback lies in the simplicity of its perception and processing as well as in its convenience and practicality. However, this 
type of digital feedback does not provide sufficient information on the required corrections and changes, does not reveal teacher's attitude to student's performance and, as a result, does not motivate, engage and involve them into revision process at all, being too monotonous and boring. In its turn, audio digital feedback mainly overcomes the above-mentioned drawbacks. However, it seems to be a bit less convenient in terms of its content analysis and further application and use. The received data allowed us to list the crucial features of a digital teacher feedback on students' translations. Survey results proved the equal value of behavioral and affective feedback aspects for translation competency acquisition by the students.

The statistical verification of the correlation of the participants' leading sensory channel (digital $(69.7 \%)$, kinesthetic $(21.21 \%)$, visual $(6.06 \%)$ and auditory $(3.03 \%)$ ), translation competency level (high (43\%), medium (36\%) and low $(21 \%)$ ) and preferred feedback modality (paper handwritten $(18.18 \%)$, written digital $(30.30 \%)$ and audio digital $(51.52 \%)$ ) revealed reliable connection between the students' translation competency level and their preferred feedback modality. On the other hand, it fixed no relation between the participants' leading sensory channel and their preferred feedback modality. Thus, the choice of feedback modality in the translation classroom should depend on the training stage and needs rather than on the trainees' individual peculiarities.

\section{REFERENCES}

Alfayyadh, Hisham M. 2016. "The Feedback Culture in Translation Education: a Comparative Exploration of Two Distinct University Translation Programs." Ph.D. diss., Kent State University.

Cavanaugh, Andrew J. and Song, Liyan. 2014. "Audio Feedback versus Written Feedback: Instructors' and Students' Perspectives." MERLOT Journal of Online Learning and Teaching 10(1):122-138.

Dalton, Zachary. 2018. "The Discourse of Written and Audio Feedback." All Master's Theses. 886. https://digitalcommons.cwu.edu/etd/886

Hattie, John and Timperley, Helen. 2007. "The power of feedback." Review of Educational Research 77(1):81-112. https://doi.org/10.3102\%2F003465430298487

Henderson, Michael and Pillips, Michael. 2015. "Video-based feedback on student assessment: scarily personal." Australasian Journal of Educational Technology 31(1):51-66.

Južnič, Tamara Mikolič. 2013. Assessment Feedback in Translator Training: A Dual Perspective. In New Horizons in Translation Research and Education, edited by N. K. Pokorn \& K. Koskinen. Joensuu: University of Eastern Finland, pp. 75-99.

Kiraly, Don. 2005. "Project-Based Learning: A Case for Situated Translation." Meta 50 (4):1098-1111. https://doi.org/10.7202/012063ar

Lim, Fei Victor and Phua, Jean. 2019. "Teaching Writing with Language Feedback Technology." Computers and Composition 54:1-13. https://doi.org/10.1016/j.compcom. 2019.102518

Lobanov, Alexandr P. 2004. Manual on the Psychology of Cognitive Processes. Minsk.

McCarthy, Josh. 2015. "Evaluating written, audio and video feedback in higher education summative assessment tasks." Issues in Educational Research 25(2):153-169.

Neunzig, Wilhelm and Tanqeiro, Helen. 2005. "Teacher Feedback in Online Education for Trainee Translators.” Meta 50(4). https://doi.org/10.7202/019873ar 
Pearson, John. 2018. "Engaging practical students through audio feedback." Practitioner Research in Higher Education Journal, 11(1):87-94.

Pietrzak, Paulina. 2014. "Towards Effective Feedback to Translation Students: Empowering through Group Revision and Evaluation." inTralinea, special issue: Challenges in Translation Pedagogy 1-6. http://www.intralinea.org/archive/article/2095

Synekop, Oksana. 2018. "Cognitive Aspect of Learning Style in Differentiated ESP Instruction for the Future IT-specialists." Advanced Education 10:40-47. https://doi.org/ $10.20535 / 2410-8286.151271$

Tsagari, Dina. 2019. "Interface between feedback, assessment and distance learning written assignments." Research Papers in Language Teaching and Learning 10(1):72-99. https://rpltl.eap.gr/current-issue/volume-10-february-2019

Voelkel, Susanne and Mello, Luciane V. 2014. "Audio Feedback - Better Feedback?" Bioscience Education, 22(1):16-30. https://doi.org/10.11120/beej.2014.00022

Washbourne, Kelly. 2014. "Beyond error marking: written corrective feedback for a dialogic pedagogy in translator training." The Interpreter and Translator Trainer 8(2):240-256. https://doi.org/10.1080/1750399X.2014.908554

Yu, Shulin, Zhang, Yiran, Zeng, Yao and Lin, Zhong. 2019. "Written Corrective Feedback Strategies in English-Chinese Translation Classrooms." Asia-Pacific Edu Res. https://doi.org/10.1007/s40299-019-00456-2

Zarei, Abbas Ali and Rezadoust, Hossein. 2020. "The Effects of Scaffolded and Unscaffolded Feedback on Speaking Anxiety and Self-efficacy." Journal of Modern Research in English Language Studies 7(4): 111-132.

Zheng, Yao, Zhong, Qiting, Yu, Shulin and Li, Xiaoohui. 2020. "Examining Students' Responses to Teacher Translation Feedback: Insights From the Perspective of Student Engagement." SAGE Open April-June:1-10. https://doi.org/10.1177/2158244020932536 